\title{
FDOPA-(18F): A PET Radiopharmaceutical Recently Registered for Diagnostic use in Countries of the European Union
}

\author{
Yanna-Marina Chevalme ${ }^{1}$, Françoise Montravers ${ }^{2}$, Jean-Philippe Vuillez ${ }^{1}$, Michel Zanca ${ }^{1}$, \\ Charles Fallais $^{1}$, Jean Oustrin ${ }^{1}$ and Jean-Noël Talbot ${ }^{1,2} *$ \\ ${ }^{1}$ AFSSAPS, St Denis; jean-noel.talbot@tnn.ap-hop-paris.fr; France. ${ }^{2}$ Service de Médecine Nucléaire; Hôpital \\ Tenon; Paris - France
}

\begin{abstract}
Positron emission tomography (PET) and its recent update PET/CT are very effective diagnostic tools for noninvasive imaging of metabolic or functional disorders in target tissues. The clinical usefulness of fluorodeoxyglucose-(18F) (FDG) has been now widely accepted. Recently, the clinical usefulness of fluoroDOPA(18F) or FDOPA, an aminoacid labelled with the same positron emitter fluorine-18, has been evaluated and recognised in France and subsequently in several EU countries. FDOPA is diagnostic PET agent, which has been used for decades in imaging the loss of dopaminergic neurons in Parkinson's disease, and more recently to detect, stage and restage neuroendocrine tumours and to search for recurrence of viable glioma tissue. The present article summarises the body of evidence that led the French Medicines Agency (AFSSAPS) to grant a marketing authorisation to IASOdopa, a commercial preparation of FDOPA. Brief case reports and figures illustrate the diagnostic performance of FDOPA PET or PET/CT in the different settings that are currently approved in oncology.
\end{abstract}

Key words: Registration of radiopharmaceuticals, PET, FDOPA, Parkinson's disease, cancer

\section{INTRODUCTION}

Positron emission tomography (PET) is a diagnostic imaging method that creates high resolution, three dimensional tomographic images of the distribution of positron emitting radionuclides labelling radiopharmaceuticals in the human body. The resulting PET images can be considered, as for any nuclear medicine technique, images of a biochemical or physiological processes ("functional images"). Positron emitting radionuclides decay results in the emission of two photons of $511 \mathrm{keV}$ in opposite directions, which can be detected simultaneously ("coincidence detection") with dedicated cameras. Then their distribution could be reconstructed to make an image using computerised tomographic reconstruction methods. Positron emitting radionuclides (carbon-11, oxygen-15, nitrogen-13, fluorine-18) have unique properties, allowing the labelling of molecules which are not possible with single-photon gamma emitters radionuclides.

For historical reasons, PET studies initially focused on the brain and the heart. Now, PET is often used in oncologic indications (Carter and Kotlyarov, 2005). This development resulted from the use of fluorodeoxyglucose-(18F) or FDG, and from the successful application of FDG-(18F) PET to a growing number of important human cancers to solve clinical problems at varying stages of

Author for correspondence 
diagnosis, staging and follow-up. However, some malignancies or neoplasms those are well differentiated or of low aggressiveness do not take-up FDG, leaving room to other PET radiopharmaceuticals in clinical oncology.

The radiopharmaceutical 6-fluoro- $\left({ }^{18} \mathrm{~F}\right)-\mathrm{L}-3,4-$ dihydroxyphenylalanine (FDOPA) has been proposed since 1986 in cognitive research on physiology and physiopathology of DOPA distribution in the human brain, and also for clinical purposes in neurology and oncology.

PET with FDOPA, provides a functional approach of pathologies, organs or tissues where enhanced intracellular transport and decarboxylation of the amino acid dihydroxyphenylalanine is the diagnostic target.

\section{Overview of the Current Application of FDOPA as a Registered Pet Radiopharmaceutical}

\section{Mode of action}

6-fluoro- $\left({ }^{18} \mathrm{~F}\right)-\mathrm{L}-3,4$-dihydroxyphenylalanine

(FDOPA) is a large, neutral amino acid that is transported into presynaptic neurons, where it is converted by the enzyme aromatic aminoacid decarboxylase [AAAD]) into fluorodopamine$\left({ }^{18} \mathrm{~F}\right)$, which subsequently enters cathecholaminestorage vesicles. 6-fluoro $\left({ }^{18} \mathrm{~F}\right)$-L-dopa crosses the blood-brain barrier; therefore, when injected into the blood stream, it reaches the dopaminergic cells in the brain and is used by the brain as a precursor for dopamine. This makes it possible to monitor intracerebral synthesis and uptake of dopamine by means of the positron-emitting 6-fluoro $\left({ }^{18} \mathrm{~F}\right)$-L3,4-dihydroxyphenylalanine (FDOPA), in conjunction with externally-placed devices suited for detection of annihilation photons, which progressively led to the most recent positron emission tomography (PET) units.

\section{Approved indications for the commercial preparation of FDOPA, IASOdopa}

\section{Neurology}

PET with IASOdopa is indicated for detecting loss of functional dopaminergic neuron terminals in the striatum. It can be used for diagnosis of Parkinson's disease and differentiation between essential tremor and parkinsonian syndromes.

\section{Oncology}

Among medical imaging modalities, PET with IASOdopa provides a functional approach of pathologies, organs or tissues where enhanced intracellular transport and decarboxylation of the amino acid dihydroxyphenylalanine is the diagnostic target. The following indications have been particularly documented:

\section{Diagnosis}

- Diagnosis and localisation of insulinomas in the case of hyperinsulinism in infants and children

- Diagnosis and localisation of glomus tumours in patients with a gene mutation of the succinate dehydrogenase $\mathrm{D}$ variant

- Localisation of pheochromocytoma and paraganglioma negative with MIBG-(123I) scintigraphy.

\section{Staging}

- Phaeochromocytoma and paraganglioma

Detection in case of reasonable suspicion of recurrences or residual disease

- Primitive brain tumours

- Phaeochromocytoma and paraganglioma negative with MIBG-(123I) scintigraphy

- Medullary thyroid cancer with elevated serum levels of calcitonin

- Well differentiated carcinoid tumours of the digestive tract

- Other endocrine digestive tumours when somatostatin receptor scintigraphy is negative

\section{Posology}

In oncology, the radioactivity usually recommended for adults is $4 \mathrm{MBq} / \mathrm{kg}$ of body mass administered by direct slow intravenous injection over approximately one minute. One half of this activity may be administered for neurological indications not requiring whole body images.

Only few clinical data are available for patients aged less than 18 years concerning safety and diagnostic efficacy of the product, except for the detection of insulinoma in infants and children. Therefore, the use in oncologic paediatrics has to be carefully weighted. In infants, the same ponderal activity (4 MBq/kg of body mass) has been proposed as in adults. 


\section{Image acquisition}

\section{Neurology}

- "dynamic" acquisition of PET images of the brain during 90 to 120 minutes right after injection

- or one "static" PET acquisition starting 90 minutes after the injection.

\section{Oncology}

To detect foci in the liver, pancreas or brain area, early "static" images can be acquired starting 5 minutes after injection, or a "dynamic" acquisition starting right after the injection during 10 minutes.

- brain tumours: "static" acquisition 10 to 30 minutes after injection.

- whole-body: images are usually acquired 60 minutes after injection

\section{Quality Aspects}

Iasodopa, the commercial preparation of FDOPA that obtained a marketing authorisation in France in November 2006 (which is currently recognised by several other EU countries), is a solution for injection.

The activity available at time of administration ranges from $0.1 \mathrm{GBq}$ to $0.8 \mathrm{GBq}$ per vial. The half-life of the radionuclide is $109.8 \mathrm{~min}$ with emission of positron radiation (Emax: 0.633 $\mathrm{MeV}$ ) followed by photon annihilation radiations of $0.511 \mathrm{MeV}$.

The composition of the product is clearly defined. The formula meets in house specifications based on the European Pharmacopoeia general monograph on Radiopharmaceuticals and USP.

The manufacturing process is run by means of an automated module and has been validated.

The quality control of the raw materials and the finished product is documented. The results comply with the European Pharmacopoeia when monographs are available and the other specific methods are validated.

\section{Drug substance}

The active ingredient of IASOdopa is the 6-fluoro(18F)-L-3,4-dihydroxyphenylalanine obtained by means of the reaction of the precursor 4,5-di[(1,1dimethylethoxycarbonyl)oxy]-N-formyl-2-

trimethylstannyl-L-phenylalanine with fluorine$\left({ }^{18} \mathrm{~F}\right)$ in freon as solvent resulting in a electrophilic exchange of the stannyl group.

The production of 6 -fluoro $\left({ }^{18} \mathrm{~F}\right)-\mathrm{L}-3,4-$ dihydroxyphenylalanine (FDOPA) is performed in two steps which occur on line: first ${ }^{18} \mathrm{~F}$ production in the cyclotron and immediately after the fully automated tracer labelling procedure. The formulation of the drug substance is an integral part of the labelling step and corresponds to the synthesis and the purification of the drug product. The pure un-dissolved drug substance cannot be separated in the manufacture process and characterised.

\section{Drug product}

The solution for injection is prepared in a shielded isolator (hot cell) and all operations of preparation and mounting of materials are conducted in a class $\mathrm{B}$ environment. The dispensing of the solution in the final container occurs after a sterile filtration on $0.22 \mu \mathrm{m}$ filter in a class A environment. The radioactive concentration of the drug product is $0.3 \mathrm{GBq}$ in $1 \mathrm{~mL}$, in glass vials of type I.

The stability studies demonstrate that the stability of the finished product is satisfactory until 16 hours after its synthesis.

\section{Non Clinical Aspects}

\section{Pharmacology}

No pharmacological activity is expected given the administered quantity of FDOPA $(0.21 \mathrm{mg} / \mathrm{kg})$.

\section{Pharmacokinetics}

The biodistribution of labelled DOPA studied in mice, dogs and humans by Mejia et al. (1991) and in rats by Bergström et al. (1997) show that there is a significant over-activity in the striatum, the kidneys and the pancreas and that the kinetics depend on the aromatic L-amino acids decarboxylase. The major part of urine activity existed as dopamine, which was eliminated by decarboxylase inhibitors such as carbidopa or benseraside.

On the basis of the reported studies of the kinetics as well as cellular metabolic bases, one may consider the use of FDOPA- $\left({ }^{18} \mathrm{~F}\right)$ for the detection and monitoring of certain tumours, which take-up and decarboxylate amine precursors.

The trials reporting the study of the kinetics clearly depicted the FDOPA- $\left({ }^{18} \mathrm{~F}\right)$ route in the brain regions and the corresponding mechanisms. They lead to the conclusion that FDOPA- $\left({ }^{18} \mathrm{~F}\right)$ is useful for evaluating the dopamine content in different tissues and its correlation with Parkinson's disease or related diseases. 


\section{Toxicology}

A single dose toxicity test was carried out with the product and revealed no deleterious effect. The absence of other toxicity data is acceptable for a product of this kind.

The toxicity data of the various potential impurities allow the conclusion that the fixed limits guarantee the toxicological safety of the product or that the corresponding compounds are not to be taken into consideration.

\section{Environmental risk assessment}

The only environmental danger is the radioactivity which, in the present case, is only to be taken into account during delivery and handling for the diagnostics, given the very short half life.

\section{Clinical Aspects}

\section{Pharmacokinetics}

The active ingredient crosses the blood-brain barrier via the large neutral amino acid transporter (NAAT), is taken-up into neurons by an active transport system, and is converted to fluorodopamine by aromatic aminoacid decarboxylase (AAAD). 6-fluoro-(18F)-L-dopa is removed according to a bi-exponential kinetic process with biological half-lives of 12 hours (67$94 \%)$ and $1.7-3.9$ hours (6-33\%). Both these half-lives appear to be age-dependent. The 18Factivity is excreted through the kidneys, $50 \%$ with a half-life of 0.7 hours and $50 \%$ with a half-life of 12 hours.

On basis of these data, a biokinetic model for 6fluoro-(18F)-L-dopa was developed. This model assumes that $100 \%$ of the $18 \mathrm{~F}$ activity is homogeneously distributed in the body and eliminated through the kidneys with biological half-lives of 1 hour $(50 \%)$ and 12 hours $(50 \%)$. This model was considered to be independent of age.

\section{Pharmacodynamics}

As for other radiopharmaceuticals for diagnosis, no pharmacodynamic effect is expected.

\section{Clinical efficacy}

FDOPA is an analogue of an aminoacid, DOPA, and is then indicated in the molecular imaging of the metabolic abnormalities of the dopaminergic pathways in neurons of the striatum. Furthermore, FDOPA has a clinical utility in neoplasms in which FDG has a poor diagnostic performance such as endocrine neoplasias-or brain tumours.

According to the marketing authorisation that was granted in November 2006 in France, the assessors sustain the request of a MRP for IASOdopa in the following clinical indications:

- in neurology, diagnosis of Parkinson's disease and differentiation between essential tremor and parkinsonian syndromes.

- in oncology, diagnosis and localisation of an insulinoma in case of hyperinsulinism in infants and children; diagnosis and localisation of glomus tumours in patients with a mutation of the succinate-deshydrogenase D subunit gene ; localisation of phaeochromocytomas and paragangliomas negative with MIBG- $\left({ }^{123} \mathrm{I}\right)$ scintigraphy; stadification of phaeochromocytomas and paragangliomas ; and detection of recurrences and residual disease of primary brain tumours, phaeochromocytomas and paragangliomas negative with MIBG- $\left({ }^{123} \mathrm{I}\right)$ scintigraphy, medullary thyroid carcinoma with an increased serum calcitonin level, and well differentiated digestive carcinoid tumours others digestive endocrine tumours when somatostatin receptor scintigraphy is negative.

\section{Neurology}

PET with IASOdopa is indicated for detecting loss of functional dopaminergic neuron terminals in the striatum. It can be used for iagnosis of Parkinson's disease and differentiation between essential tremor and parkinsonian syndromes

In neurology, the main point is that parkinsonian subjects with normal dopaminergic imaging do not have evidence of classical PD or an atypical parkinsonian syndrome. Considering that about 20 $\%$ of patients diagnosed with PD are discovered false-diagnosed after post mortem examination, this aspect constitutes a key challenge in helping clinicians to ascertain positive diagnosis of PD. The typical pattern of FDOPA uptake in PD is a severe impairment of uptake in the posterior part of the putamen (less than half of normal), less impairment in the anterior putamen (ca. 60\% of normal) and a relatively spared FDOPA uptake in the caudate (ca. 85\% of normal) (Brooks et al. , 1990; Kuwabara et al. , 1995; Lee et al. 2000). Thus IASOdopa, the use of which must be helpful in the diagnosis and the subsequent management of potential PD patients, fully deserves a marketing authorisation to diagnose PD in the relatively rare clinically difficult cases. 
The pathophysiological mechanism explaining the reduction of FDOPA uptake in the striatum in PD is still controversial. Kinetic analysis led Kuwabara et al. (1995) to conclude that, in PD, there is no change in the blood to brain FDOPA transport, but the activity of aromatic aminoacid decarboxylase (AAAD) is differentially reduced in the subdivisions of the striatum.

Furthermore, is has been shown that the striatal to occipital ratio which can be used to assess the diagnosis of parkinsonism is, in routine examinations, a far easier and thus robust way to evaluate the pathology level than the FDOPA influx constant, thus favouring a possible large diffusion of the technique.

In a study of Ghaemi et al. (2001), data from 12 early Parkinson's disease (PD) and 9 advanced PD patients were compared with those from 13 agematched healthy controls. The study has shown a significant increase of FDOPA influx and relative activity in the pineal gland of PD patients. Additionally, significant correlations were found between influx and the Hoehn and Yahr (Handy) scores, and between the relative FDOPA activity and the parameters disease duration, Handy disease score and Unified Parkinson's Disease Rating Scale (UPDRS). The result may be explained as a compensatory upregulation of monoaminergic transmitter systems outside the basal ganglia even if increased FDOPA uptake in the pineal gland may only reflect pineal dysfunction in PD patients. The purpose of the paper of Lee et al. (2000) was to compare striatal uptake of tracers for the vesicular monoamine transporter DAT and AAAD in idiopathic Parkinson diseased (IPD) patients versus control individuals. It shows AAAD activity upregulation but also DAT downregulation in IPD patients. One could conclude, at a first sight, that this underestimation of presynaptic dopaminergic neuronal loss gives advantage to DATSCAN, compared to FDOPA, in terms of detection sensitivity of IPD patients, particularly at the early beginning of the pathology. But, similarly to FDOPA, FPCIT overestimates terminal loss in IPD owing to compensatory down-regulation. Indeed, Eshuis et al. (2006) has shown, with a Bland-Altman plot of FPCIT SPECT and FDOPA PET that, at low uptake values (in advanced disease), FDOPA is more sensitive while, at higher uptake values (in early disease), FPCIT is more sensitive; Eshuis globally concluded, as did Ishikawa et al. (1996), that, for both tracers, the striatal to occipital ratio values discriminated PD patients from controls, with a similar accuracy. This is in accordance with the general consensus asserting that the currently available data do not suggest a significant advantage, in terms of clinical usefulness, of PET over SPECT (Ishikawa et al. , 1996; Brücke et al. , 2000; Tatsch, 2002; Eshuis et al. , 2006). In the same study of Eshuis (2006), performed with both FPCIT SPECT and FDOPA PET in patients with de novo PD and advanced PD, sensitivity and specificity values were calculated after performing receiver operator characteristic (ROC) analysis: sensitivity of FPCIT SPECT for discrimination between de novo and advanced PD patients was 0.88 , while specificity was 0.70 ; for FDOPA PET, sensitivity for discrimination between the two patient groups was 0.88 and specificity, 0.77, without significant differences between PET and SPECT. When putaminal values were used instead of striatal ones, sensitivity and specificity of both methods remained approximately the same (sensitivity and specificity for FPCIT $=0.77$ and 0.71 respectively vs. $=0.76$ and 0.85 respectively for FDOPA). Thus, one has to consider that FDOPA is a PET marker, with the recognised superiority of PET detection (higher spatial resolution, direct 3D acquisition with attenuation and diffusion corrections, and more accurate quantification); in comparison with SPECT used for DATSCAN. Furthermore, due to the sufficient half-life of $18 \mathrm{~F}$ and a better access to PET centres in Europe, FDOPA will be accessible to most neurologists with an important point to consider: FDOPA uptake is an objective biological gauge of the dopaminergic pathway and represents a rational criterion to ascertain the diagnosis of PD. Indeed, Eckert et al. (2007) has shown that, "of a group of 185 patients with clinical signs of PD who underwent FDOPA PET imaging for diagnostic confirmation, 27 patients $(15 \%)$ had quantitatively normal examinations. None of these patients developed clinical signs of classical PD or of an atypical parkinsonian syndrome at a follow-up visit conducted 3 years after imaging, thus suggesting that parkinsonian subjects with normal dopaminergic imaging do not have evidence of classical PD or an atypical parkinsonian syndrome". Considering that about $20 \%$ of patients diagnosed with PD are discovered falsediagnosed after post mortem examination, this capacity of FDOPA PET constitutes, in our 
opinion, key evidence in helping clinicians to ascertain positive diagnosis of PD.

\section{Oncology : diagnosis}

- Diagnosis and localisation of insulinomas in the case of hyperinsulinism in infants and children (Fig.1).

Insulinomas are a difficult medical challenge; indeed, although generally benign, there are responsible for severe hypoglycaemia, and must be surgically removed, while diffuse hyperplasia of beta cells must be managed in another way. Diagnosis of hyperinsulinism is easy to confirm using insulin and C-peptide assays in serum, but localisation of the potential tumour, which is as a rule less than $2 \mathrm{~cm}$ in diameter, is most often difficult. Pentetreotide-(indium 111) scintigraphy is not performing, and echoendoscopy must be performed and is not always contributive.

FDOPA PET is a very effective non-invasive method for the detection of the focal form of hyperinsulinism in infants, with a very high accuracy. A major impact on decision-making and on guiding surgery can be derived from several recent and concordant studies.

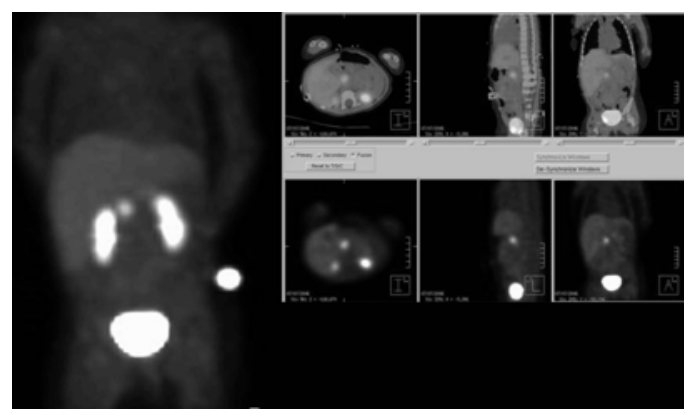

Figure 1 - This infant was referred to hôpital Tenon for FDOPA (IASOdopa $\left.{ }^{\circledR}\right)$ PET/CT in search for an insulinoma that could explain his crises of hypoglycemia. A clear focus was seen in the head of the pancreas, resected surgically and the crises disappeared

This is of a major importance for the infant's management: hyperinsulinism with focal lesion can revert by selective surgical resection in contrast to the diffuse form, which requires subtotal pancreatectomy when resistant to medical treatment.

The diagnostic accuracy of FDOPA PET does appear near $100 \%$ in all studies (of course they concern few patients, as hyperinsulinemia is infrequent), enabling the diagnosis of focal adenomatosis with a very high sensitivity, the diagnosis of diffuse involvement as well, and more over it allows very well distinguishing focal adenomatosis from diffuse hyperplasia.

These data were recently confirmed in a series of 24 infants with medically unresponsive hyperinsulinism (Hardy et al. 2007): the diagnosis of local or diffuse hyperinsulinism was correct in 23 of 24 cases (96\%). FDOPA PET identified focal areas of high uptake of radiopharmaceutical in 11 patients. Pathology results confirmed that all 11 had focal adenomatosis, and the locations of these lesions matched the areas of increased
FDOPA uptake on the PET scans in all of the cases.

Moreover, a recent paper confirmed the interest of FDOPA in this clinical setting also in adults patients (Kauhanen et al. 2007).

- Diagnosis and localisation of glomus tumours in patients with a gene mutation of the succinate dehydrogenase $\mathrm{D}$ variant

FDOPA PET proved to be effective for the detection of phaeochromocytoma or paraganglioma or chemodectoma, suspected on biological evidences, such as SDHD mutation or "familial paraganglioma syndrome type 1" (PGL1). In those rather rare cases, it could be more cost effective to perform FDOPA PET as the first step examination without prior MIBG-(123I) scintigraphy, since PET imaging is of superior quality and FDOPA detected much more foci and was more accurate than MIBG-(123I).

Actually, FDOPA PET was proposed as early as 2003 by Hoegerle et al. for the systematic detection of a tumour of chromaffin cells origin, chemodectoma also called glomus tumour, in 
patients with proven mutations of the succinate dehydrogenase subunit D (SDHD) gene, predisposing to the development of glomus tumours and other paragangliomas (PGL1). FDOPA PET and MRI were performed in 10 consecutive such patients. Both imaging modalities were assessed under blinded conditions by two experienced specialists in nuclear medicine (PET) and diagnostic radiology (MRI). Afterwards the results were compared. A total of 15 foci (4 solitary and 4 multifocal tumours, the latter including 11 lesions) were detected by FDOPA PET. Under blinded conditions, FDOPA PET and MRI revealed full agreement in 7 patients, partial agreement in 2 and complete disagreement in 1 . Eleven of the 15 presumed tumours diagnosed by FDOPA PET were confirmed by MRI. The correlation of FDOPA PET and MRI confirmed 3 further lesions previously only detected by PET.

Novosel et al. (2004) subsequently reported the case of a 13-year-old boy with an atypical manifestation of a multilocular paraganglioma (PGL1), in whom FDOPA PET was successful to detect extensive spread of the disease (both adrenal glands, paravertebral paraganglioma and bilateral cervical glomus tumours), as hypertensive crises persisted after surgical extirpation of a left sided abdominal paraganglioma. Recently the team in Freiburg (Brink et al. 2006) reported another case of a 12 year-old boy with this mutation leading to PGL1. FDOPA PET allowed the localisation of 4 unknown lesions (right adrenal, paravertebral abdominal, and one in each side of the neck). This illustrates the utility of FDOPA PET prior to surgery in those patients with the SDHD mutation.

This indication is only sustained by short series and case reports, as its deals with a very rare pathology. However, considering the clear impact on management for those patients, we think that there is a clinical utility of FDOPA in this rare indication.

- Localisation of pheochromocytoma and paraganglioma negative with MIBG- $\left({ }^{123} \mathrm{I}\right)$ scintigraphy

Phaeochromocytomas and paragangliomas are rare tumours, which are generally revealed by enhanced secretion of catecholamines and biological features. Thus the clinical and therapeutic problem is to localise the tumour. Very often morphological imaging is inefficient and scintigraphy demonstrates a strong additional value. Considering the sometimes limited sensitivity of MIBG, and of other radiopharmaceuticals as well (i.e. FDG, pentetreotide-(In111)), it appears of great interest to use FDOPA PET which demonstrated higher diagnostic performances. Thus despite the limited number of patients and of published clinical studies, FDOPA deserves to be registered and used in this indication. Obviously, FDOPA could not be a standard first line examination, as some studies found a $100 \%$ sensitivity and positive predictive value with combination of MIBG and MRI (Lumachi et al. , 2006). But it could be very useful in rare cases nor detected or not correctly staged with conventional imaging, including specific scintigraphies.

FDOPA-PET imaging in phaeochromocytoma has been proposed by Hoegerle in 2002. FDOPA PET and magnetic resonance (MR) imaging were performed in 14 consecutive patients suspected of having phaeochromocytomas (5 sporadic, 9 with von Hippel-Lindau disease); MIBG scintigraphy was performed in 12 of them. The individual imaging findings were assessed in consensus by specialists in nuclear medicine and radiologists blinded to the results of the other methods. The findings of the functional imaging methods were compared with those of MR imaging, the reference standard. Histologic verification could be obtained in 8 patients with 9 tumours. Seventeen phaeochromocytomas (11 solitary, 3 bifocal; 14 adrenal, 3 extra-adrenal) were detected with MR imaging. FDOPA-PET and MR imaging had concordant results in all 17 tumours. In contrast, MIBG scintigraphy had false-negative results in 4 patients with 3 adrenal tumours smaller than $2 \mathrm{~cm}$ and 1 extra-adrenal tumour with a diameter of 3.6 $\mathrm{cm}$. On the basis of these data, sensitivities of $100 \%$ for FDOPA-PET and of $71 \%$ for MIBG scintigraphy were calculated. Specificity was $100 \%$ for both procedures.

The team in Freiburg that proposed this use of FDOPA-PET also investigated paragangliomas (Brink et al. , 2005). A reported specificity of $99 \%$ and a cumulative sensitivity of about $90 \%$ still make MIBG-(123I) a relevant first line nuclear imaging method. However, they reported that "FDOPA-PET seems to be a very promising procedure, which offers higher accuracy".

Recently, a supportive study has been presented (Helisch et al. , 2006). In 25 patients with a suspicion of phaeochromocytoma on biological evidences, 20 lesions were visualised on both FDOPA and MIBG imaging, 21 on FDOPA PET 
only and 2 on MIBG scintigraphy only. Superiority of FDOPA was most obvious when thoracic paragangliomas were finally discovered : 19/19 were depicted on FDOPA PET vs. 1/19 on MIBG scintigraphy. By just considering lesions assessed with histology, FDOPA sensitivity was 95\% (41/43) vs. 51\% (22/43) for MIBG. Authors conclude that FDOPA PET should be part of the pretherapeutic staging work-up in case of pheochromocytoma or paraganglioma. This is concordant with the experience of Montravers et al in series of 11 patients (Montravers et al. , 2006a): FDOPA detected a greater number of foci than MIBG; a change in patient management was induced in 2 patients among 8 .

\section{Oncology : staging}

- Phaeochromocytoma and paraganglioma All previous considerations and conclusions concerning the detection and localisation of phaeochromocytomas and paragangliomas could be applied to metastatic workup as well, and the product could be proposed for staging of these tumours. The above reported result not only refers to the localisation of the primary tumour but also to the detection of the metastatic lesions.

\section{Oncology: detection in case of reasonable suspicion of recurrences or residual disease}

- Primitive brain tumours (Fig.2).

In primary brain tumours, imaging of amino acid transport in brain lesions is more sensitive than 2fluoro-(18F)-deoxyglucose (FDG) PET. First because there is a high physiological uptake of FDG by the normal cortex of the brain and second due to the well-known correlation between FDG uptake and tumour grade. Low-grade gliomas, as well as some grade III gliomas, either cannot be distinguished from the background in normal brain tissue or present as cold lesions in relation to the uptake of the normal cortex, thus severely hampering the use of FDG PET in follow-up.

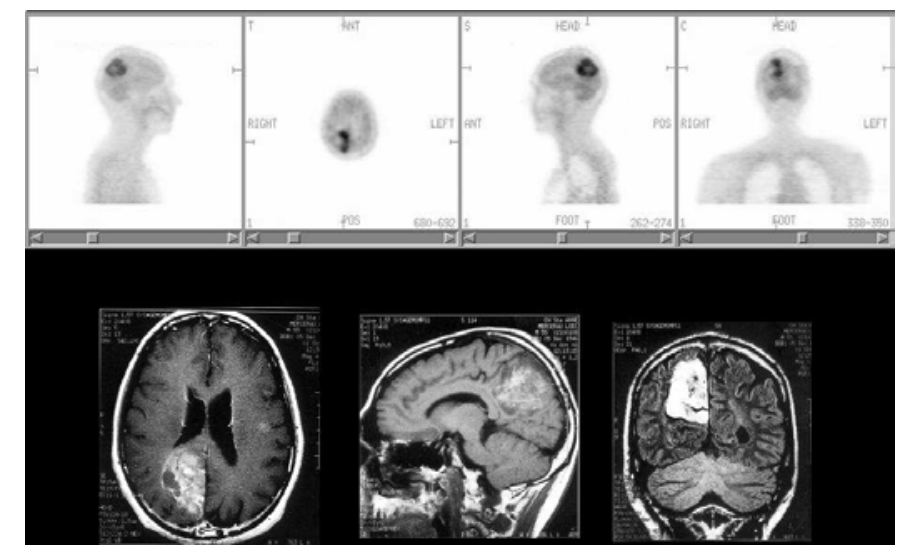

Figure 2 - Grade III oligo-astrocytoma treated by radiotherapy 13 years ago. MRI showed a questionable increase in the size of the residual mass. The occipital recurrence was clearly seen on FDOPA (IASOdopa®) PET performed at hôpital Tenon. Surgery was then indicated and the recurrence was confirmed by histology

The first description of imaging a brain tumour with FDOPA-PET is a case report by Heiss et al. as early as 1996. Then Becherer (2003) demonstrated the similar performances of FDOPA PET in this setting, in comparison with methionine-(11C) (MET), in series of 20 patients with known supratentorial brain lesions. FDOPA(18F) appeared to be an accurate surrogate for methionine-(11C) in imaging amino acid transport in malignant cerebral lesions, for the purpose of visualisation of viable tumour tissue. It combines the good physical properties of $18 \mathrm{~F}$ with the pharmacological properties of an aminoacid. That was more recently confirmed in a larger studies (Chen et al. ,2006): a head to head study demonstrated a significantly greater sensitivity of FDOPA over FDG that is a registered comparator for the detection of recurrence of high grade gliomas.

- Phaeochromocytoma and paraganglioma negative with MIBG-(123I) scintigraphy(Fig. 3) 
As previously mentioned, all above considerations and conclusions concerning the detection and localisation of phaeochromocytomas and paragangliomas, using fluoroDOPA in case of negative MIBG-(123I) scintigraphy could be applied to metastatic workup, and, in the same way, for the detection and localisation of recurrences and residual disease as well.
Medullary thyroid cancer with elevated serum levels of calcitonin (Fig. 4).

FDOPA-PET appears to be a useful supplement to morphological diagnostic imaging, including FDG-PET, in medullary thyroid cancer (MCT), improving lymph node staging and enabling a more specific diagnosis of primary tumour (in presurgical staging) and of local and distant recurrences.
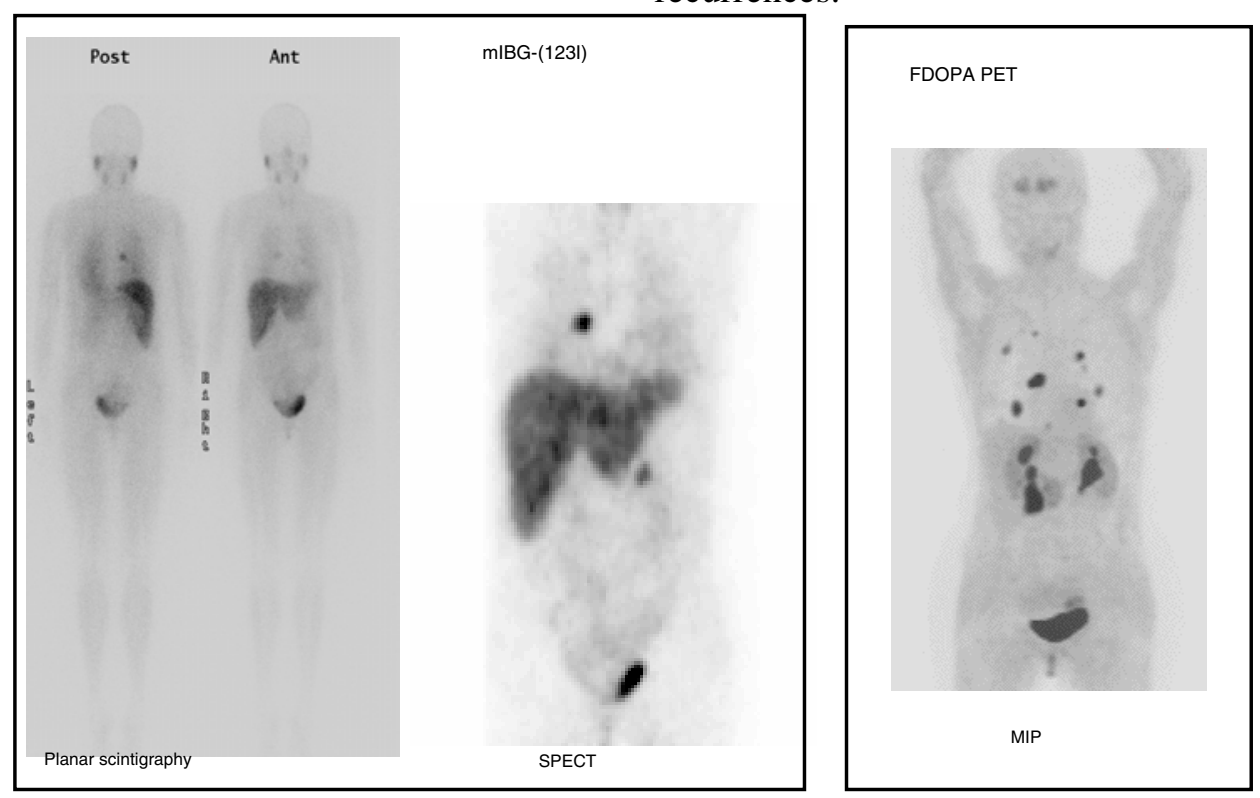

Figure 3 - Patient operated 4 years before from a phaeochromocytoma of the left adrenal gland. An increase in urinary cathecholamine excretion motivated MIBG-(123I) scintigraphy and SPECT that showed one isolated lesion in the mediastinum. FDOPA (IASOdopa $\left.{ }^{\circledR}\right)$ PET, performed at hôpital Tenon for re-staging, confirmed the lesion in the mediastinum but also showed 6 infracentimetric pulmonary or pleural nodules. Thoracic CT and MR were then requested an concluded to benign vascular images in the lungs ; the lesion in the mediastinum was thus resected and actually corresponded to recurrent phaeochromocytoma. However, during follow-up, urinary cathecholamine excretion re-increased and the pulmonary lesions seen on the re-staging FDOPA PET became obvious

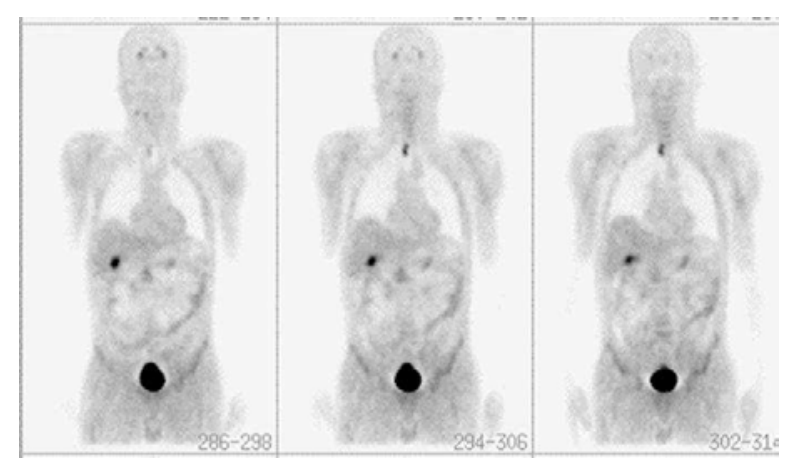

Figure 4 - Occult recurrence of a medullary thyroid cancer operated 5 years before, suspected on rising serum calcitonin levels (up to $900 \mathrm{pg} / \mathrm{mL}$ ), that remained unexplained. The 
patient was referred to Hôpital Tenon where FDOPA (IASOdopa®) PET/CT discovered right cervical lymph nodes, prevertebral lymph nodes at the level of the right pulmonary apex and foci in the skull

FDOPA-PET has been shown to be able to detect occult recurrences, i.e. rising calcitonin or CEA serum levels without localisation of the recurrence after conventional work-up. This setting may become a major indication since calcitonin assay is very sensitive and specific to detect the existence of a recurrence, sometimes of a very small size, but therapeutic management needs the recurrence to be localised.

Overall, from a total of 86 patients in this rare cancer, evidence has been provided that FDOPA is superior to the two comparators of nuclear imaging, FDG and pentetreotide, and it has added value to morphologic imaging. It showed a significant impact and patient management. A recent series in 15 patients (Beuthien-Baumann et al. 2007) confirmed the role of FDOPA in biologic recurrence of MCT and the authors concluded: "Neuroendocrine uptake of FDOPA seems to be more specific than FDG uptake for the detection of metastases of MTC'.

- Well differentiated carcinoid tumours of the digestive tract (Fig. 5)

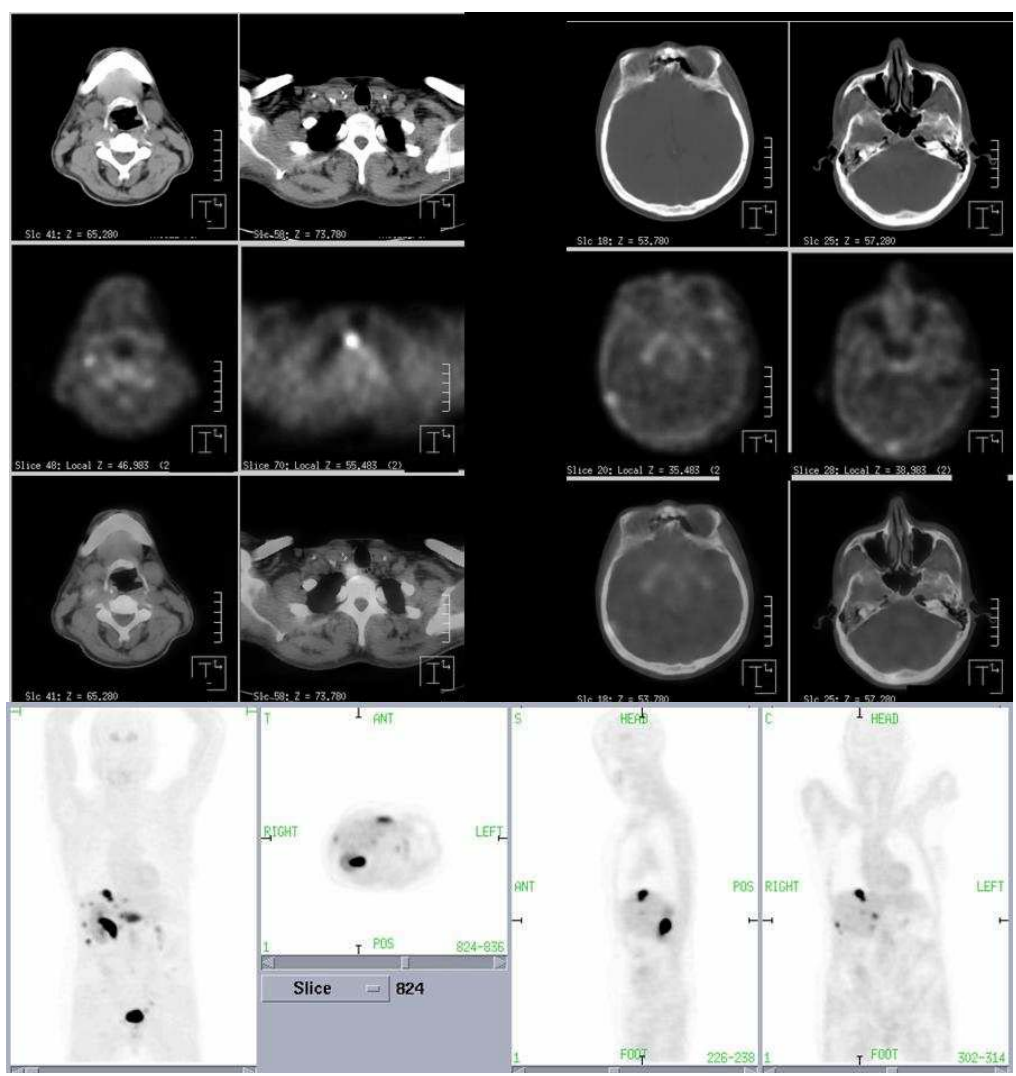

Figure 5 - Carcinoid tumour of the midgut already operated 3 times in the past.. Rising serum levels of chromogranine A. CT only showed 3 liver nodules $(5 \mathrm{~mm}, 6 \mathrm{~mm}$ et $18 \mathrm{~mm})$ and an infiltration of the perirectal fat ; pentetreotide-(111In) SPECT was negative. Patient was referred to hôpital Tenon where FDOPA (IASOdopa ${ }^{\circledR}$ ) PET/CT showed active secondary lesions in the liver and in the surrounding peritoneum, which were subsequently confirmed by post surgical histology

- Other endocrine digestive tumours when somatostatin receptor scintigraphy is negative.
Endocrine tumours of the gastrointestinal tract are relatively uncommon neoplasms, characterised by a great heterogeneity. Carcinoid tumours are 
usually classified-according to embryonic origininto foregut, midgut, and hindgut, a classification that is still relevant because these different tumours have different tendencies to spread out and different symptomatology.

Endocrine pancreatic tumours (EPT) include insulinomas, gastrinomas, VIPomas, glucagonomas and somatostatinomas. In malignant tumours, mixed syndromes are common due to multiple hormone production from the tumours. In about one-third of EPT, the patients do not present with any hormone-related symptoms, and these tumours are referred as non-functioning tumours. EPT can also be part of multiple endocrine neoplasia-1 (MEN-1) syndromes, in which multiple pancreatic tumours are almost always found. Again, imaging is often disappointing in the work-up of these tumours, including FDG-PET and pentetreotide scintigraphy as well that morphological imaging. The use of FDOPA-PET is based on the capacity of endocrine tumour cells to take-up, decarboxylate and store aminoacids, such as DOPA.

The initial publications of Hoegerle et al. (2001b) and Becherer et al. (2004) has shown a potential clinical usefulness of FDOPA PET in this setting. More recently, the study by Montravers et al. (2006b) included 33 patients who were parted into two groups, 19 patients with carcinoid tumours and 14 patients with other endocrine digestive tumours, all had both FDOPA PET and pentetreotide scintigraphy (SRS). FDOPA PET confirmed to be more sensitive than SRS in case of differentiated carcinoid tumour $(93 \%$ vs. $81 \%$ on per patient basis). However, in other endocrine digestive tumours, FDOPA PET was clearly less sensitive ( $25 \%$ vs. $75 \%$ respectively). This finding can be related to the data of Ahlström et al. (1995), quoted above, and using carbon-11 labelled DOPA and PET in endocrine pancreatic tumours. Only half of these tumours (11/22) were detected by DOPA-(11C) PET, which matches the $50 \%$ accuracy of FDOPA-PET Montravers et al. (2006a) observed in non-carcinoid endocrine tumours. These data have been further confirmed in the recent paper of Koopmans et al. (2006).

In conclusion, since pentetreotide-(111In) is expensive (more than twice the price of a dose of FDG-(18F) in France), it is recommended to perform a precise histological determination of the endocrine digestive tumour, in order to choose the best radiopharmaceutical (among SRS, FDG and FDOPA that are currently registered in France), on a case by case basis: the first line examination in poorly differentiated endocrine digestive tumours should be FDG-(18F) PET, the first line examination in carcinoid tumours should be FDOPA-(18F) PET, while pentetrotide-(111In) is still the best registered radiopharmaceutical for non-carcinoids digestive endocrine tumours, in particular those of the pancreas.

\section{Clinical safety}

The tolerance of FDOPA-(18F) is very good. An irradiation of the patient is expected, far below the level of non-stochastic effects, the radiation dose values being similar to that of FDG-(18F) i.e. around 5- $7 \mathrm{mSv}$ for PET alone.

Only one case of the induction of a carcinoid crisis has been reported (Koopmans et al. , 2005), after rapid injection of the radiopharmaceutical. This should be avoided, and a slow injection over $1 \mathrm{~min}$ is advised, the more so as the final preparation is still acid and some patient complained from a very transient pain on the site of injection at a more rapid rate. Using this mode of administration, the Paris-Tenon team reported no serious effect in 170 patients with endocrine tumours (Nataf et al. , 2006), which was confirmed by the team in Groningen that originally reported the adverse event. However, attention should be paid to the fact that non-labelled carrier is also present and injected together with FDOPA-(18F). Therefore, the specific activity of an in-house preparation of FDOPA should be carefully monitored prior to injection, to avoid agonist clinical effects.

\section{CONCLUSIONS}

FDOPA that has been used for neurologic PET examinations for many years has now demonstrated its clinical usefulness to diagnose and monitor patients with a large and precise scope of pathologies. It is important to stress that those pathologies are not covered by FDG which is the only other PET radiopharmaceutical currently registered in the European Union for diagnosis. It is also important to guarantee a GMP production of FDOPA according to the monography in USP or very recently in Eur Pharm, the sterility, apyrogenicity, radiochemical purity and specific activity of which is controlled. 


\section{RESUMO}

Tomografia por emissão de positrons (PET) e sua recente atualização PET/CT são ferramentas de diagnóstico muito eficientes para imagens não invasivas de desordens metabólicas ou funcionais em tecido alvo. A utilidade clínica da fluordesoxiglicose-(18F)(FDG) tem sido agora largamente aceita. Recentemente, a utilidade clinica de fluoroDOPA-(18F) ou FDOPA, um aminoácido marcado com o mesmo emissor de pósitron, flúor-18, tem sido avaliado e reconhecido na França e subsequentemente em alguns países da União Européia. FDOPA é o radiofármaco para diagnóstico em PET, o qual tem sido usado por décadas para obtenção de imagens da perda de neurônios dopaminérgicos na doença de Parkinson $\mathrm{e}$, mais recentemente, para identificar inicialmente o estágio e a reavaliação de tumores neuroendócrinos e para a pesquisa da recorrência de glioma viável. $\mathrm{O}$ presente artigo resume o conjunto de evidências que levarão a Agência Médica Francesa (AFSSAPS) garantir uma autorização de divulgação da IASOdopa, uma preparação comercial da FDOPA. O relato breve de caso e figuras que ilustram um padrão de imagem de diagnóstico usando FDOPA em PET ou PET/CT em diferentes configurações que são aceitas em oncologia.

\section{REFERENCES}

Ahlström, H., Eriksson, B., Bergström, M., Bjurling, P., Langstrom, B., Oberg, K. (1995), Pancreatic neuroendocrine tumors: diagnosis with PET. Radiology 195, 333-337.

Ambrosini, V., Tomassetti, P., Rubello, D., Campana, D., Nanni, C., Castellucci, P., Farsad, M., Montini, G., Al-Nahhas, A., Franchi, R., Fanti, S. (2007), Role of 18F-DOPA PET/CT imaging in the management of patients with $111 \mathrm{In}$-pentreotide negative GEP tumours. Nucl. Med. Commun., 28, 473-477.

Becherer, A., Karanikas, G., Szabo, M., Zettinig, G., Asenbaum, S., Marosi, C., Henk, C., Wunderbaldinger P, Czech T, Wadsak W, Kletter K. (2003), Brain tumour imaging with PET : a comparison between [18F] fluorodopa and [11C] methionine. Eur. J. Nucl. Med. Mol. Imaging, 30, 1561-1567.

Becherer, A., Szabo, M., Karanikas, G., Wunderbaldinger, P., Angelberger, P., Raderer, M., Kurtaran, A., Dudczak, R., Kletter, K. (2004),
Imaging of advanced neuroendocrine tumors with 18F-FDOPA PET. J. Nucl. Med., 45, 1161-1167.

Beheshti, M., Poecher, S., Heinisch, M. et al. Assessment of medullary thyroid carcinoma with 18F-DOPA PET/CT in comparison with 18F-FDG PET/CT. EANM annual meeting, Eur. J. Nucl. Med., 2006, S131, abstract $\mathrm{n}^{\circ} 227$.

Bergstrom, M., Lu, L., Marquez, M., Fasth, K., Bjurling, P., Watanabe, Y., Eriksson, B., Langstrom, B. (1997), Modulation of organ uptake of 11Clabelled L-DOPA. Nucl. Med. Biol., 24, 15-19.

Beuthien-Baumann, B., Strumpf, A., Zessin, J., Bredow, J., Kotzerke, J. (2007), Diagnostic impact of PET with (18)F-FDG, (18)F-DOPA and 3-O-methyl6 -[(18)F]fluoro-DOPA in recurrent or metastatic medullary thyroid carcinoma. Eur. J. Nucl. Med. Mol. Imaging, in press.

Brink, I., Hoegerle, S., Klish, J., Bley, T. A. (2005), Imaging of pheochromocytoma and paraganglioma. Fam. Câncer, 4, 61-68.

Brink, I., Schaefer, O., Walz, M., Neumann, H. (2006), Fluorine-18 DOPA PET imaging of paraganglioma syndrome. Clin. Nucl. Med., 31, 39-41.

Brooks, D., Ibanez, V., Sawle, G., Quinn, N., Lees, A. J., Mathias, C., Bannister, R., Marsden, C., Frackowiak, R. (1990), Differing patterns of striatal 18F-dopa uptake in Parkinson's disease, multiple system atrophy, and progressive supranuclear palsy. Ann. Neurol., 28, 547-555.

Brücke, T., Djamshidian, S., Bencsits, G., Pirker, W., Asenbaum, S., Podreka, I. (2000), SPECT and PET imaging of the dopaminergic system in Parkinson's disease. J. Neurol., 247, IV/2-IV/7.

Carter, K., Kotlyarov, E. (2005), PET and PET/CT imaging for the earliest detection and treatment of colorectal carcinoma. Braz. Arch. Biol. Technol., 48 (Suplement),169-174.

Chen, W., Silverman, D., Delaloye, S., Czernin, J., Kamdar, N., Pope, W., Satyamurthy, N., Schiepers, C., Cloughesy, T. (2006), 18F-FDOPA PET imaging of brain tumors: comparison study with 18F-FDG PET and evaluation of diagnostic accuracy. J. Nucl. Med., 47,904-911.

Eckert, T., Feigin, A., Lewis, D., Dhawan, V., Frucht, S., Eidelberg, D. (2007), Regional Metabolic Changes in Parkinsonian Patients with Normal Dopaminergic Imaging. Mov. Disord.., 22, 167-173.

Eshuis, S., Maguire, R., Leenders, K., Jonkman, S., Jager, P. (2006), Comparison of FP-CIT SPECT with F-DOPA PET in patients with de novo and advanced Parkinson 's disease. Eur. J. Nucl. Med. Mol. Imaging, 33, 200-209.

Ghaemi, M., Rudolf, J., Hilker, R., Herholtz, K., Heiss, D. (2001), Increased pineal Fdopa uptake is related to severity of Parkinson's disease - A PET study, $J$. Pineal Res., 30, 213-219.

Hardy, O. T., Hernandez-Pampaloni, M., Saffer, J., Suchi, M., Ruchelli, E., Zhuang, H., Ganguly, A., 
Freifelder, R., Adzick, N., Alavi, A., Stanley, C. (2007), Diagnosis and localization of focal congenital hyperinsulinism by $18 \mathrm{~F}$-fluorodopa PET scan. $J$. Pediatr., 150, 140-145.

Heiss, W. D., Wienhard, K., Wagner, R., Lanfermann, H., Thiel, A., Herholz, K., Pietrzyk, U. (1996), FDopa as an amino acid tracer to detect brain tumors. J. Nucl. Med., 37, 1180-1182.

Helisch, A., Fottner, C., Schreckenberger, M., Weber M, Bartenstein P. (2206), Pre-therapeutic localisation of multifocal pheochromocytomas and paragangliomas: superiority of F18-DOPA PET vs. iodine-123-MIBG scintigraphy. SNM annual meeting, 228.

Hoegerle, S., Altehoefer, C., Ghanem, N., Brink, I., Moser, E., Nitzsche E. (2001), 18F-DOPA positron emission tomography for tumour detection in patients with medullary thyroid carcinoma and elevated calcitonin levels. Eur. J. Nucl., Med., 28, 64-71.

Hoegerle, S., Altehoefer, C., Ghanem, N., Koehler, G., Waller, C., Scheruebl, H., Moser, E., Nitzsche, E. (2001), Whole-body 18F DOPA PET for detection of gastrointestinal carcinoid tumors. Radiology, 220, 373-380.

Hoegerle, S., Nitzsche, E., Altehoefer, C., Ghanem, N., Manz, T., Brink, I., Reincke, M., Moser, E., Neumann, H. (2002), Pheochromocytomas : detection with ${ }^{18} \mathrm{~F}$ DOPA whole-body PET - initial results. Radiology, 222, 507-512.

Hoegerle, S., Ghanem, N., Altehoefer, C., Schipper, J., Brink, I., Moser, E., Neumann, H. P. (2003), 18FDOPA positron emission tomography for the detection of glomus tumours. Eur. J. Nucl. Med. Mol. Imaging, 30, 689-694.

Ishikawa, T., Dhawan, V., Kazumata, K., Chaly, T., Mandel, F., Neumeyer, J. (1996), Comparative nigrostriatal dopaminergic imaging with iodine-123$\beta C I T-F P / S P E C T$ and fluorine-18-FDOPA/PET. $J$. Nucl. Med., 37, 1760-1765.

Kauhanen, S., Seppänen, M., Minn, H., Gullichsen, H., Salonen, R., Alanen A. (2007), Fluorine-18-Ldihydroxyphenylalanine (18F-DOPA) positron emission tomography as a tool to localize an insulinoma or beta-cell hyperplasia in adult patients. J. Clin. Endocrinol. Metab., 92, 1237-1244.

Koopmans, K., Brouwers, A., De Hooge M., Van der Horst-Schrivers, A., Kema, I., Wolffenbuttel, B., De Vries, E., Jager, P. (2005), Carcinoid crisis after injection of 6-18F-fluorodihydroxyphenylalanine in a patient with metastatic carcinoid. J. Nucl. Med., 46, 1240-1243.

Koopmans, K., De Vries, E., Kema, I., Elsinga, P., Neels, O., Sluiter, W., van der Horst-Schrivers, A., Jager, P. (2006), Staging of carcinoid tumours with
18F-DOPA PET : a prospective diagnostic study. Lancet Oncol., 7, 790-792.

Kuwabara, H., Cumming, P., Yasuhara, Y., Leger, G. C., Guttman, M., Diksic, M., Evans, A., Gjedde, A. (1995), Regional striatal DOPA transport and decarboxylase activity in Parkinson's disease. $J$. Nucl. Med., 36, 1226-1231.

Lee, C., Samii, A., Sossi, V., Ruth, T., Schulzer, M., Holden, J., Wudel, J., Pal, P., de la Fuente-Fernandez, R., Calne, D., Stoessl, A. (2000), In vivo positron emission tomographic evidence for compensatory changes in presynaptic dopaminergic nerve terminals in Parkinson's disease. Ann. Neurol., 47, 493-503.

Lumachi, F., Tregnaghi, A., Zucchetta, P., Cristina Marzola, M., Cecchin, D., Grassetto, G., Bui, F. (2006), Sensitivity and positive predictive value of CT, MRI and 123I-MIBG scintigraphy in localizing pheochromocytomas: a prospective study. Nucl. Med. Commun., 27, 583-587.

Mejia, A., Nakamura, T., Itoh, M., Hatazawa, J., Ishiwata, K., Ido, T., Matsumoto, M., Watabe, H., Watanuki, S., Seo, S. (1991), Absorbed dose estimates in positron emission tomography studies based on the administration of 18F-labeled radiopharmaceuticals. J. Radiat. Res. (Tokyo), 32, 243-261.

Montravers, F., Grahek, D., Kerrou, K., Grahek, D., Gutman, F., Leverger, G., Talbot, J. (2006a), Impact de la TEP à la fluoro-(18F)-dihydroxyphénylalanine (FDOPA) sur l'attitude thérapeutique en cas de tumeur endocrine: tumeur digestive, cancer médullaire de la thyroïde ou phéochromocytome. Med. Nucl., 50, 383-390.

Montravers, F., Grahek, D., Kerrou, K., Ruszniewski, P., de Beco, V., Aide, N., Gutman, F., Grange, J., Lotz, J., Talbot, J. (2006b), Can fluorodihydroxyphenylalanine (FDOPA) positron emission tomography replace somatostatin receptor scintigraphy in patients with digestive endocrine tumors ? J. Nucl. Med., 47,1455-1462.

Mottaghy, F., Karges, W., Zeich, K. et al. Detection of recurrent medullary thyroid carcinoma (MTC) by F18-DOPA PET/CT. SNM annual meeting 2006, $\mathrm{N}^{\circ}$ 472.

Nataf, V., Balard, M., De Beco, V., Kerrou, K., Gutman, F., Grahek, D., Montravers, F., Talbot, J. (2006), Safety of 18F-DOPA injection for PET of carcinoid tumor. J. Nucl. Med., 47, 1732.

Novosel, A., Heger, A., Lohse, P., Schmidt, H. (2004), Multiple pheochromocytomas and paragangliomas in a young patient carrying a SDHD gene mutation. Eur. J. Pediatr., 163, 701-703.

Otonkoski, T., Näntö-Salonen, K., Seppänen, M., Veijola, R., Huopio, H., Hussain, K., Tapanainen, P.,

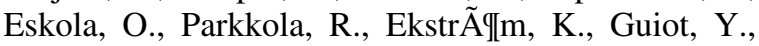
Rahier, J., Laakso, M., Rintala, R., Nuutila, P., Minn, H. (2006), Noninvasive diagnosis of focal 
hyperinsulinism of infancy with [18F]-DOPA positron emission tomography. Diabetes, 55,13-18.

Ribeiro, M.., De Lonlay, P., Delzescaux, T., Boddaert, N., Jaubert, F., Bourgeois, S., Dolle, F., NihoulFekete, C., Syrota, A., Brunelle, F. (2005), Characterization of hyperinsulinism in infancy assessed with PET and 18-Fluoro-L-DOPA. J. Nucl. Med., 46,560-566.

Santiago-Ribeiro, M., De Lonlay, P., Bourgeois, B. et al. Diagnostic value of [18F]fluoro-L-DOPA PET in the differential diagnosis between focal and diffuse hyperinsulinism : report of a 40 children study. SNM annual meeting, 2006, $\mathrm{N}^{\circ} 409$.

Tatsch, K. (2002), Can SPET imaging of dopamine uptake sites replace PET imaging in Parkinson's disease. Eur. J. Nucl. Med., 29, 711-714.

Wintering, N., O'Rourke, S., Saffer, J. et al. Diagnostic imaging using 18F-labeled L-fluoro-DOPA PET scans assists in treatment planning for pediatric patients with hyperinsulinism. SNM annual meeting $2006, \mathrm{~N}^{\circ} 410$. 